\title{
Spatial updating in blind and sighted people
}

\author{
MARK HOLLINS and ELIZABETH K. KELLEY \\ University of North Carolina, Chapel Hill, North Carolina
}

\begin{abstract}
Four experiments were carried out to determine the accuracy with which early-blind and blindfolded sighted subjects could indicate the remembered positions of objects. Experiment 1 showed that the position of a single object can be recalled, usually with little decline in accuracy, for at least $24 \mathrm{~min}$. Experiment 2 indicated that the recalled position of one object exerts little, if any, influence on the recalled position of an adjacent object. Experiments 3 and 4 dealt with "updating," the ability to examine objects from one location, then walk to a new location, and from there indicate the positions of the objects. Both groups of subjects demonstrated good updating ability when the method of responding was to replace objects in their original positions; when required to point to objects, however, blind subjects made significantly larger errors than sighted subjects. The pattern of errors suggested distorted recall of the stimulus field rather than an inability to transform spatial information.
\end{abstract}

On tests of spatial ability, particularly those that depend on the manipulation of mental representations, subjects who became blind at an early age often perform at a lower level than blindfolded, sighted persons (Marmor \& $\mathrm{Za}-$ back, 1976; Rieser, Guth, \& Hill, 1982, 1986; Witkin, Birnbaum, Lomonaco, Lehr, \& Herman, 1968; Worchel, 1951). Extensive experimental analysis is often needed for a complete understanding of such differences, however, for it is not always clear to what cognitive, perceptual, or motor factors a given difference in performance is attributable. For example, data that seem to indicate a relative slowness of mental rotation in blind people (Marmor \& Zaback, 1976) may instead reflect only a greater stability of their haptic frame of reference (Carpenter \& Eisenberg, 1978; Hollins, 1986).

One important spatial ability on which early-blind and blindfolded sighted people have been reported to differ is "spatial updating" (Rieser et al., 1982), or sensitivity to "changes in the structure of their perspectives" (Rieser et al., 1986). These terms refer to a person's ability to alter his or her ideas as to where objects in the environment are located, with respect to him- or herself, when moving among them. For example, suppose a blind man were facing a tree, and then made a $180^{\circ}$ turn in place; if he subsequently knew, without depending on new sensory information, that the tree was behind him, he could be said to have accomplished a certain amount of spatial updating.

This research was funded by a grant from the University Research Council. We are grateful to Eugene Long and Tom Wallsten for valuable discussion, and to John Rieser and an anonymous reviewer for their comments on an earlier draft of the manuscript. A preliminary report of these experiments was presented at the annual meeting of the Psychonomic Society, Boston, November 1985, and appeared in abstract form in the Bulletin of the Psychonomic Society, 23, 278. Correspondence should be addressed to the first author at the Department of Psychology, Davie Hall, University of North Carolina, Chapel Hill, NC 27514.
The importance of updating was emphasized by Rieser et al. (1982), who distinguished this component of spatial cognition from knowledge of layout. They suggested that a person may acquire a firm knowledge of where objects are located with respect to each other, and to the self, and yet have difficulty in modifying that knowledge to take his or her own movements into account. To assess the dependence of updating ability on prior visual experience, they asked early-blind, late-blind, and blindfolded sighted individuals to enter an unfamiliar room, to let themselves be guided from a starting location to a number of objects, one at a time, and then to demonstrate their knowledge of the positions of the objects by pointing to them from the starting location; after this, the subjects were again guided to the location of one of the objects, and then asked to point from there to the remaining objects. Although the three groups of subjects were comparable in the accuracy of their points from the starting location, they differed dramatically in their ability to point from the second location. The sighted subjects were the most accurate, closely followed by the late-blind subjects; the pointing errors of the early-blind subjects, in contrast, were nearly three times as great from the new location as they had been at the starting location. Comparable data were obtained in an extensive follow-up study by the same authors (Rieser et al., 1986). The authors attributed the large errors of the early-blind group to a relative inability on their part to keep track of their changing relationships to their environment during locomotion.

The present study was designed to test the generality of the findings of Rieser et al. $(1982,1986)$ in two ways. First, a smaller experimental area was used: a tabletop, on which subjects were able to locate objects rapidly and without assistance. Because there is evidence that different factors affect the ability to process spatial information in near and far space (Warren, Anooshian, \& Bollinger, 1973), a determination of whether updating ability follows the same rules in the two types of situations would 
help to define the range of conditions over which Rieser et al.'s (1982) analysis applies.

As a second way of exploring the generality of Rieser et al.'s $(1982$, 1986) findings, we used an additional type of response: subjects were asked not only to point to objects, but also to replace objects in their proper positions on the table. It was hoped that, through this use of converging operations, the present study would be able to provide new information regarding the nature of the difficulty in updating experienced by early-blind subjects.

Four experiments were carried out. Experiments 1 and 2 explored the feasibility of requiring subjects to recall object positions for as long as $24 \mathrm{~min}$, and assessed the vulnerability of memory for a test object to interference from haptic inspection of a nearby distractor object. These preliminary experiments laid the groundwork for an investigation of updating, using two types of responses: pointing (Experiment 3) and replacing (Experiment 4).

\section{METHOD}

\section{Subjects}

Blind subjects. There were 5 blind subjects, 3 females and 2 males, ranging in age from 19 to 36 years. Two had been born blind due to prenatal glaucoma, and another 2 had lost their sight shortly after birth as a result of retrolental fibroplasia. These 4 subjects were totally blind. A 5th subject had been born with impaired vision due to prenatal glaucoma; his vision gradually grew worse until, by age 7 , he was left with light perception only, which he still retains. This subject may thus be considered intermediate between early- and late-blind. His data, however, were quite consistent with those of the other blind subjects, closely resembling the group medians. All 5 subjects participated in each of the four experiments.

Sighted subjects. There were 6 sighted subjects, 4 females and 2 males, ranging in age from 19 to 21 years. All had normal vision. One (female) participated in Experiments 1 and 2 only, while another (male) participated in Experiments 3 and 4 only. The other subjects participated in all four experiments. Thus, there were 5 sighted subjects in each experiment.

All subjects were naive with respect to the purposes of the study.

\section{Apparatus}

The apparatus was a 92-cm-high table made from a disk of Plexiglas, $91.4 \mathrm{~cm}$ in diameter, that was securely mounted on a steel barrel. Sheets of plastic, ruled in millimeters, were attached to the underside of the tabletop so that positions on the table could be specified. Two small threaded holes in the tabletop, near the edge and separated from one another by $90^{\circ}$, allowed pointer-protractor assemblies to be attached (Figure 1). The pointers were $1-\mathrm{cm}$-wide Plexiglas strips, tapered to a blunt point at the front, which could be swiveled about a pivot at the back end. The pointer was pivoted at the center of the protractor, and the direction in which the pointer was aimed could be read from the scale with an accuracy of about $10^{\prime}$ arc. For most of an experimental session, only one or the other of the pointer-protractor assemblies was attached to the table. As far as the experimenters could determine from careful and continuous observation, no subject ever discovered, while standing at one protractor, the hole in the table marking the position of the (absent) other protractor.

The subject's task was to learn, and later to recall, the location of small objects placed on the table. These were everyday objects, no more than $2 \mathrm{~cm}$ in size, such as an aspirin, a pebble, and a ball of aluminum foil. The same set of objects and positions was used

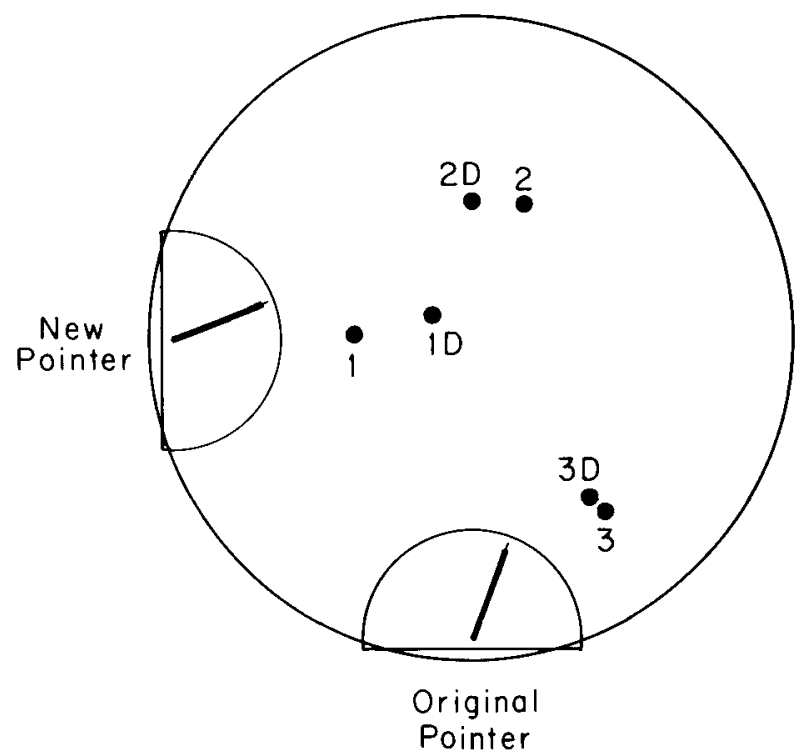

Figure 1. Diagram of the apparatus, showing the original pointerprotractor assembly (used in all four experiments), and the "new" one (used in Experiments 3 and 4 only). Only one assembly was present on the table at any given time, except when the subject was in the process of moving from one response station to the other. Also shown are the locations $(1,2$, and 3) of the test objects used in Experiments 1 and 2 and of their respective distractors (1D, 2D, and 3D) in Experiment 2.

for all subjects. Object locations for Experiments 3 and 4 were selected randomly, their $x$ and $y$ coordinates (in centimeters) being obtained from a table of random numbers. For the learning phase of each run, the objects were affixed to the table with sticky wax; before the testing phase began, the wax was completely removed. The learning phase always took place with the subject standing behind a particular one of the pointers; this will be referred to as the "original" pointer (or original response station); the other pointer, located $90^{\circ}$ clockwise from this, will be called the "new" pointer (response station).

\section{Procedure}

Before entering the experimental room, which they had never seen, sighted subjects were blindfolded with a pair of flexible safety goggles covered with electrician's tape and adhesive-backed velour paper. The goggles were strapped firmly to the subject's head, so that the flange was pressed against the skin all around; in response to questioning, the subjects said they could see nothing, a statement that was confirmed by the surprise all but 1 of them showed at the (small) size of the apparatus when the goggles were removed at the end of the last session. Blind subjects also wore the goggles to control for any distracting effects of the goggles themselves.

Each subject was tested individually in a series of 90 -min sessions separated from one another by at least $24 \mathrm{~h}$. Three sessions were needed for Experiments 1 and 2 together; an additional three sessions were needed for Experiments 3 and 4. A 15-min break was given in the middle of each session.

Preliminary experiments. The purpose of Experiment 1 was to familiarize the subjects with the apparatus, and to determine whether their memory for the location of an object would decline or change appreciably over the course of a run. In each of the three runs making up this experiment, the subject was asked to stand behind the original pointer, to examine, by touch, an object attached to the table with wax, and then to point to it intermittently over the course of $25 \mathrm{~min}$. 
Once the testing phase had begun, the subject was asked not to reach out onto the table beyond the confines of the protractor, a restriction that was also observed in Experiments 2 and 3. After each response, the experimenter recorded the direction of the pointer, and then moved it a variable, but usually substantial, distance to the left or right. Blocks of five trials each (beginning at $0,6,12$, 18 , and 24 min following the removal of the stimulus) were interspersed with short breaks during which the subject sat at a nearby desk and assembled objects out of interlocking plastic bricks.

The first two such runs (separated by a break) made up the first session; the third was carried out at the beginning of the second session. A different object in a different position was used in each run.

The purpose of Experiment 2 was to learn whether memory for the location of one object is subject to any spatial distortion when a second object is introduced into the field. Since later experiments were to make use of groups of objects, it was thought advisable to learn beforehand, insofar as possible, whether interactions would occur between remembered locations. The procedure for this experiment was identical to that of Experiment 1, except that at $11 \mathrm{~min}$ following the removal of the test stimulus, a distractor object, clearly different from the test object, was attached to the table, and the subject was asked to examine this new object and remember its location. In fact, the subject was never tested on his or her memory for the position of the distractor object; instead, the issue was whether the introduction of the distractor would influence the remembered location of the test object in subsequent trials within the run. The test objects used in the three runs of Experiment 2 were different from those in Experiment 1, but their respective locations were the same as in the first experiment, in order to allow the closest possible comparison of pointing responses obtained in runs with and without a distractor. All stimulus positions are specified in Figure 1. This experiment occupied half of the second and all of the third session for each subject; thus, the two runs employing a given test position never took place on the same day.

Main experiments. The purpose of Experiment 3 was to measure the ability of the subjects to take changes in their own position into account when pointing to a given set of objects from two different directions. The design of the experiment was for the subjects to learn the positions of objects while standing at the original pointing station, and then to point to those locations from each pointing station. The experiment began with the subject's being asked to locate and learn the position of a particular object, named by the experimenter. This was the only stimulus object present on the table. After the subject had located and examined it, the experimenter removed it and attached the next object in the series to (a different position on) the table. After all five objects had been individually presented, the subject was asked whether he or she wanted to examine any of them a second time; the subjects usually asked for one or two of the objects to be presented again. Testing was not begun until the subject expressed confidence in his or her knowledge of the objects' positions. It is important to emphasize that all learning took place while the subject was standing at the original pointer. The "new" pointer-protractor assembly was not present on the table at this time; it was brought and attached to the table only when it was needed during testing.

In the testing phase, which immediately followed the learning phase, responses from the two pointers were obtained in one of two orders. Subjects either made a series of responses from the original pointer and then moved to the new pointer for a second series (type A runs) or they went to the new pointer at the beginning of testing, made a series of responses, and then returned to the original pointer for a final series (type B runs). Runs of the two types were counterbalanced for each subject, the order being ABBA for 3 subjects in each group, and BAAB for the others. Experiment 3 took two sessions, with two runs, separated by a break, making up each session.

For the first series of trials within a run, the subject was instructed to stand at a specific pointer and to use it to point to each of the objects as it was named by the experimenter. Only this one pointerprotractor assembly was present on the table. The objects were named five times each, in random order, subject to the constraint that each be named once before any was named twice, and so on. After recording each response, the experimenter turned the pointer a variable distance to the right or left.

Upon completion of these 25 trials, the experimenter attached the other pointer to the table, and the subject was asked to walk alongside the table until he or she reached this second pointer. The subject was encouraged to maintain contact with the tabletop while walking and, on reaching his or her destination, to examine both pointers simultaneously in order to obtain a clear impression of their relative locations. After this, the pointer at which the subject had already been tested was removed from the table. These same steps were followed whenever the subject went from one pointer to the other. Twenty-five trials were then carried out from the second pointer, the same objects being named five times each according to a new random order. This completed a run.

The overall plan of Experiment 4 was similar to that of Experiment 3 , except that subjects responded during the testing phase by replacing objects on the table rather than pointing to them. The experiment began with subjects standing at the original response station and examining five objects, which were attached to the table one at a time, just as in Experiment 3. These were new objects, in new positions. Once the subject expressed confidence in his or her knowledge of the objects' positions, the testing phase began.

During testing, the experimenter handed the subject a single object, named it, and asked the subject to replace it in its original position on the table. After the response had been made, the experimenter recorded the $x$ and $y$ coordinates of the object's position, removed the object from the table, and handed the subject the next object in the series. Replacement trials were somewhat more time consuming than pointing trials, so runs were limited to three trials with each of the five objects from each response station. They were arranged in random order, subject to the same constraint used in the previous experiment. After an initial series of trials at one response station, the subject walked alongside the table until he or she reached the other response station, following the procedure adopted in Experiment 3, and then a second series of object replacements was made from this location.

Within a given run, the subjects were tested either first at the original response station and then at the new response station or the reverse. The single session devoted to Experiment 4 comprised two runs, one of each type. The type A run came first for 3 subjects in each group and second for the remaining subjects.

Considering Experiments 3 and 4 together, there were a total of six runs, in each of which 5 objects were employed. Each of these 30 objects was in a different position, with these positions the same for all 10 subjects. Thus, for every object, the median response of all subjects within a group could be calculated.

The order of Experiments 3 and 4 was counterbalanced across subjects: The single replacing session preceded the two pointing sessions for 2 subjects in each group, followed pointing for 2 others, and was executed between the two pointing sessions for the 5th subject in each group.

\section{RESULTS}

\section{Preliminary Experiments}

Experiment 1. The data of Experiment 1, in which subjects pointed repeatedly at a given object over the course of a run, are illustrated by the open symbols in Figure 2. Triangles represent data for the blind subjects, and circles represent data for the sighted subjects. Because a subject's five responses within a block of trials were usually in close agreement (standard deviations typically 


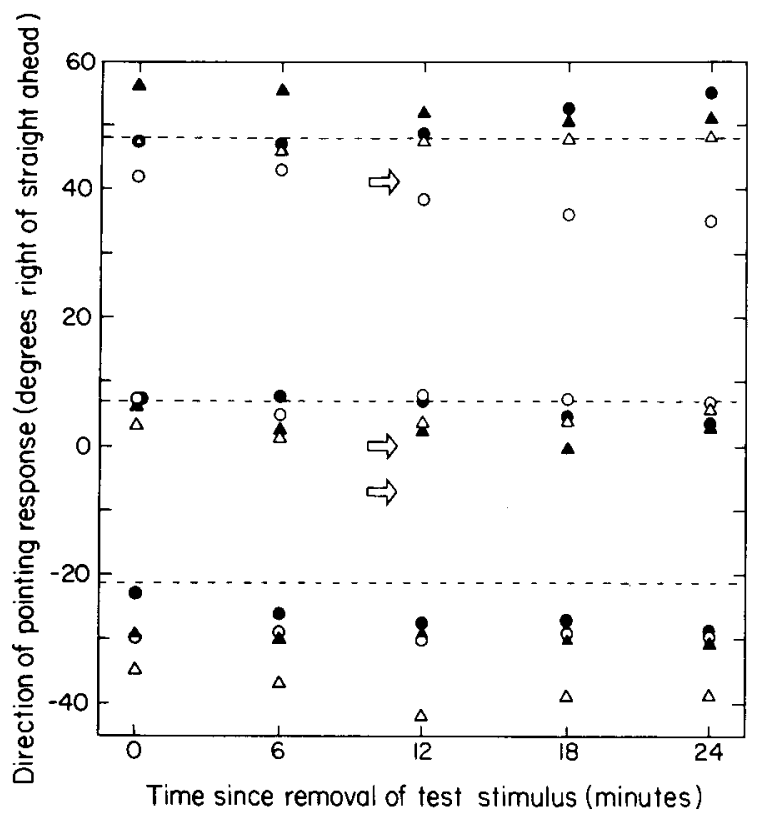

Figure 2. Median directions of pointing in the preliminary experiments, for sighted (circles) and blind (triangles) subjects. Data in the lower, middle, and upper regions of the graph refer to runs using test objects located at Positions 1, 2, and 3 (see Figure 1), respectively. That the presentation of a distractor at $11 \mathrm{~min}$ had no consistent effect on the data can be seen by comparing the results of Experiment 2 (filled symbols) with those of Experiment 1 (open symbols), in which no distractor was used. The dashed lines indicate the direction from the center of the protractor to the actual locations of the test objects; the lower, middle, and upper arrows show the direction and time of presentation of distractors indicated in Figure 1 as 1D, 2D, and 3D, respectively.

being on the order of $2^{\circ}-4^{\circ}$ ), the mean of these five values was determined and used in subsequent calculations. To combine data across subjects, however, the median of these means was taken, since it is not clear that the abilities being measured are normally or even unimodally distributed within the blind population. Each symbol in Figure 2 represents the across-subject median direction of pointing at a particular object, for the series of responses beginning at the time indicated on the abscissa. Data for the three objects used in the different runs of the experiment are shown in the lower, middle, and upper parts of the figure, respectively. The dashed horizontal lines show the actual direction in which each object was located: roughly $21^{\circ}$ to the left of straight ahead for object $1,7^{\circ}$ right for object 2 , and $48^{\circ}$ right for object 3 .

The data indicate that subjects recalled, and were able to point in, the general direction of the objects. That is, they pointed roughly straight ahead for object 2 and to the left and right of straight ahead for objects 1 and 3, respectively. Accuracy was not high, however: median errors were approximately $8.2^{\circ}$ for the sighted subjects and $5.5^{\circ}$ for the blind subjects. This difference between the groups was not statistically significant (two-tailed Mann-Whitney $U$ test, $p>.05$ ). Individual subjects' responses sometimes drifted substantially over the course of a run, and in some cases even the group data for a particular object show such drifts. Drifts toward the correct direction were almost as common as drifts away from it, however, so that there was no consistent tendency for errors to increase over the course of a run.

Experiment 1 showed that our subjects were able to point in the approximate direction of objects for at least $24 \mathrm{~min}$, and probably longer, following their last contact with the object. This result made clear the feasibility of more elaborate experiments ( 3 and 4 ) in which the testing period was, by necessity, of about this duration. Temporal drifts in the Experiment 1 data, however, suggested the desirability of counterbalancing the conditions of such later experiments, a precautionary step that was incorporated into their design.

Experiment 2. The question addressed by this experiment was whether haptic examination of one object would influence the subject's memory for the location of another object through interference, field effects, or any other mechanism. The procedure was identical to that of Experiment 1 , except that during the 12th minute following the removal of the test stimulus, the subject was instructed to inspect and recall the position of a distractor object fastened to the table a short distance from the position previously occupied by the test object.

The results of Experiment 2 are shown by the filled symbols in Figure 2, where once again triangles show the data of the blind subjects and circles, the data of the sighted subjects. The lower, middle, and upper arrows indicate, respectively, the angular position (see Figure 1) and time of presentation of the first, second, and third distractor objects. Any effect of a distractor would presumably show up as a measurable shift in subsequent responses to the test object, especially for the block of trials beginning at $12 \mathrm{~min}$, when compared with the data obtained earlier in the run. However, no consistent shift, either toward or away from the position of the distractor, is seen at 12 min for any of the objects. This result implies that interactions among memories of the locations of different objects are not a major factor in this situation, and thus supports the feasibility of using multiple objects in the main experiments.

\section{Main Experiments}

Magnitude of error. In Experiment 3, the ability of subjects to update their knowledge of spatial layout was assessed by requiring them to point at a given object from two different response stations. A subject's five pointing responses to a given object from a given response station were averaged, and this mean was used in subsequent calculations that combined data across subjects or objects. Errors were expressed as the absolute value of the angular difference between the subject's mean response and the correct orientation of the pointer.

In Experiment 4, a subject's three replacement responses with a given object and from a given response sta- 
tion were-like the pointing responses-usually in close agreement. The $x$ coordinates of the three responses were averaged, as were their $y$ coordinates, to give a mean replacement position. To facilitate comparison between the pointing and replacing data, replacement locations were expressed in terms of their radial direction from the pivot of the pointer-protractor assembly at which the subject stood while responding. As in Experiment 3, error scores were then obtained by calculating the absolute value of the difference between the subject's mean response and the direction in which the object had originally been located.

The sizes of errors in all conditions of Experiments 3 and 4 are shown in Figure 3. The height of each bar shows the median error for a particular condition, combined first across objects for each subject and then across subjects within each group. Open bars indicate errors made from the original response station, and hatched bars show errors made from the new response station.

The open bars on the left side of the histogram indicate that the two groups of subjects pointed fairly accurately from the original response station; from the new station, however, performance was not so good. The difference in median pointing errors between the two response stations was of moderate size in the case of the sighted subjects and very large in the case of the blind subjects. Both differences were statistically significant (one-tailed Wilcoxon tests, $p=.031$ in both cases). Direct comparisons of the two groups, using one-tailed Mann-Whitney $U$ tests, showed that the median pointing errors of the blind subjects were significantly larger than those of the sighted subjects at the new response station $(p=.016)$ but not at the old $(p>.05)$.
These data are in close agreement with those of Rieser et al. $(1982,1986)$, and extend the generality of their findings by showing that, on a pointing task, early-blind subjects demonstrate little updating ability, even when tested on a stimulus field smaller by an order of magnitude than the ones used in their experiments.

However, the replacing errors from Experiment 4, shown on the right in Figure 3, show a different pattern. Here median performance was excellent not only from the original pointer, but from the new one as well. The difference in accuracy between the two response stations was insignificant (one-tailed Wilcoxon test, $p>.05$ ) for both the sighted and blind subjects. Moreover, the median errors of the blind subjects were not significantly different from those of the sighted subjects at either response station (one-tailed Mann-Whitney $U$ tests, $p>.05)$. Thus, when asked to use replacement as a method of responding, both blind and sighted subjects demonstrate considerable updating skill.

To ensure that expressing replacement responses in angular form did not cause the loss of important information regarding response accuracy, statistical tests were also carried out on the linear errors of the subjects' replacements, both in the table's $x$ dimension (horizontal in Figure 1) and in the $y$ dimension (vertical in Figure 1). In neither dimension were the errors of the blind subjects significantly larger at the new response station than at the original station; the same was true for the sighted subjects (one-tailed Wilcoxon tests, $p>.05$ in all cases). When the errors of the blind subjects were compared with those of the sighted subjects using Mann-Whitney $U$ tests, replacement responses of the former group were found

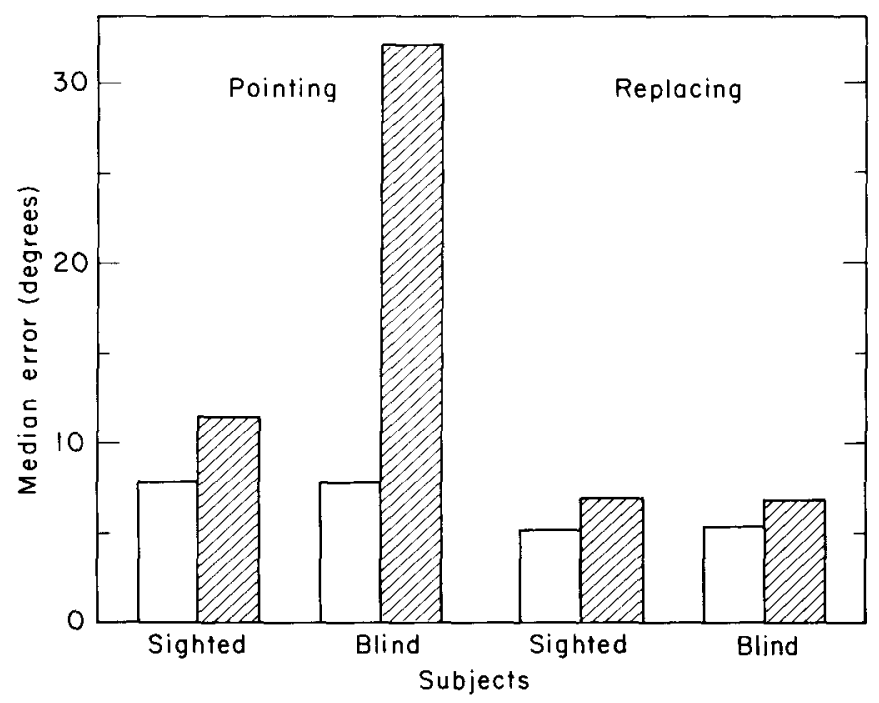

Figure 3. Summary of the data of Experiments 3 (left) and 4 (right), with median errors in responding from the original pointer (open bars) and from the new pointer (hatched bars) shown separately for the sighted and blind subjects. These values were obtained by determining the median, across subjects within a group, of the individual subjects' median errors for a particular condition. 
to be marginally less accurate in the $x$ dimension than those of the latter group, at the original station ( $p=.048$, onetailed). However, there was no statistical difference between the groups in $x$-dimension errors when subjects stood at the new station, or in $y$-dimension errors at either station ( $p>.05$ in all three cases). Thus, the analysis of dimensional errors confirms the overall picture of replacement accuracy obtained when responses are expressed in angular form.

Taken together, the data of Experiments 3 and 4 indicate that, when they move from place to place, many blind subjects, like sighted ones, can keep track of their surroundings, but are unable to demonstrate this updating ability if required to use pointing as a response mode. The reasons for this powerful effect of response mode cannot, however, be determined by considering just the magnitude of errors: it is also necessary to examine the types of errors made by the subjects.

Patterns of error. To determine whether there were any consistent differences in the types of errors made by the two groups of subjects or with the two response modes, median responses for each object were calculated. These are shown in Figures 4 through 7, which are similar to one another in format.

Plotted in Figure 4 are the replacement responses of the sighted subjects. The open symbols represent responses made from the original response station, and the filled symbols represent replacements made from the new station. On the ordinate is plotted the direction from the hub of the protractor to the spot on the table where

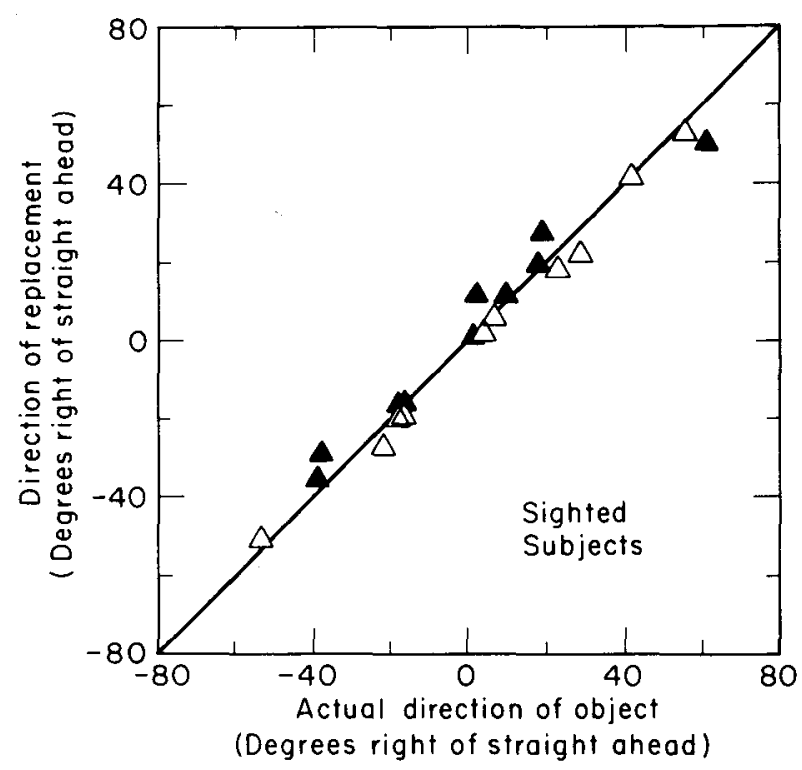

Figure 4. Replacing responses of the sighted subjects. Direction of response, defined as the direction from the center of the protractor at which the subject stood while responding to the location in which he/she replaced each object, is plotted as a function of the direction from the same protractor to the original location of the object. Open symbols show responses made from the original station; filled symbols show responses from the new station.

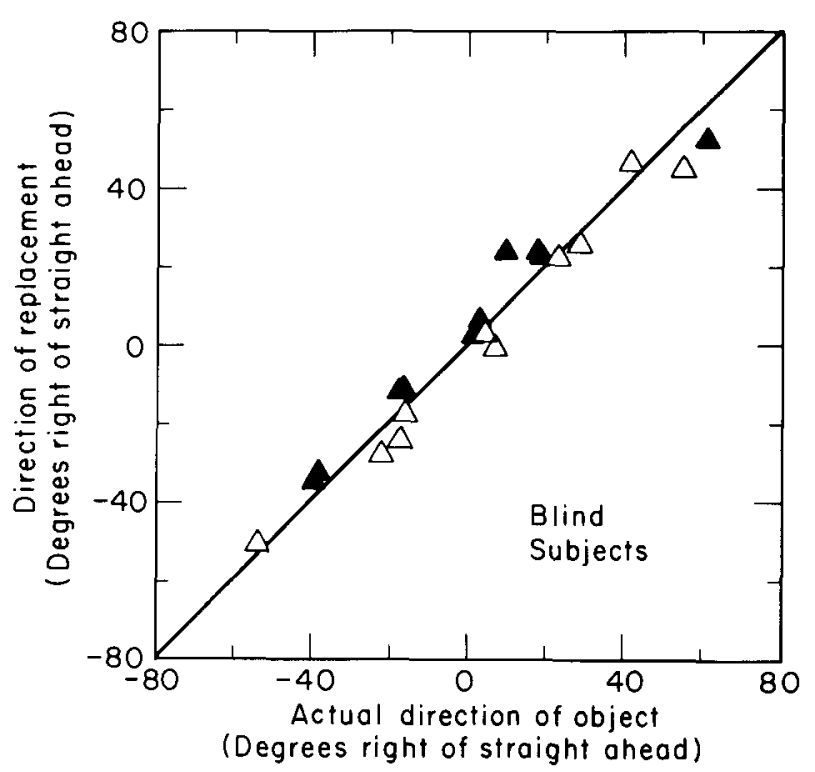

Figure 5. Replacing responses of the blind subjects. Conventions as in Figure 4.

the subject placed each object. Directions are expressed with respect to straight ahead (i.e., toward the center of the table), from whichever pointing station he or she occupied at a given time. Abscissa values are the directions to the position in which each test object had been located during the learning phase-in other words, the directions in which the subject "ought" to have responded.

The proximity of most of the measurements to the diagonal indicates that these subjects had fairly accurate knowledge of where most of the objects had been. They were nearly as accurate from the new response station as they were from the original one, a result that shows them to be capable of updating their knowledge of spatial relationships to take into account their own altered locations.

Figure 5 shows the median replacement data for the blind subjects. As in the previous figure, filled and open symbols lie about equally close to the diagonal, a result that indicates good spatial updating ability.

Median pointing responses for the sighted subjects are plotted in Figure 6. The responses made from the original pointer were quite accurate, but there is a suggestion of somewhat larger errors in pointing from the new station. As stated in connection with Figure 3, this difference in error magnitude between the two pointers is statistically significant. Moreover, there is some consistency in the errors made from the new pointer: most of the filled circles lie above the diagonal, showing that from this station the sighted subjects tended to point slightly to the right of the correct direction. On the whole, however, performance is fairly good.

The pointing data for the blind subjects, plotted in Figure 7 , contrast sharply with those of the sighted subjects. Here errors from the new pointer are very large: most of the filled circles lie well above the diagonal. The blind subjects thus tended to point far to the right of the 


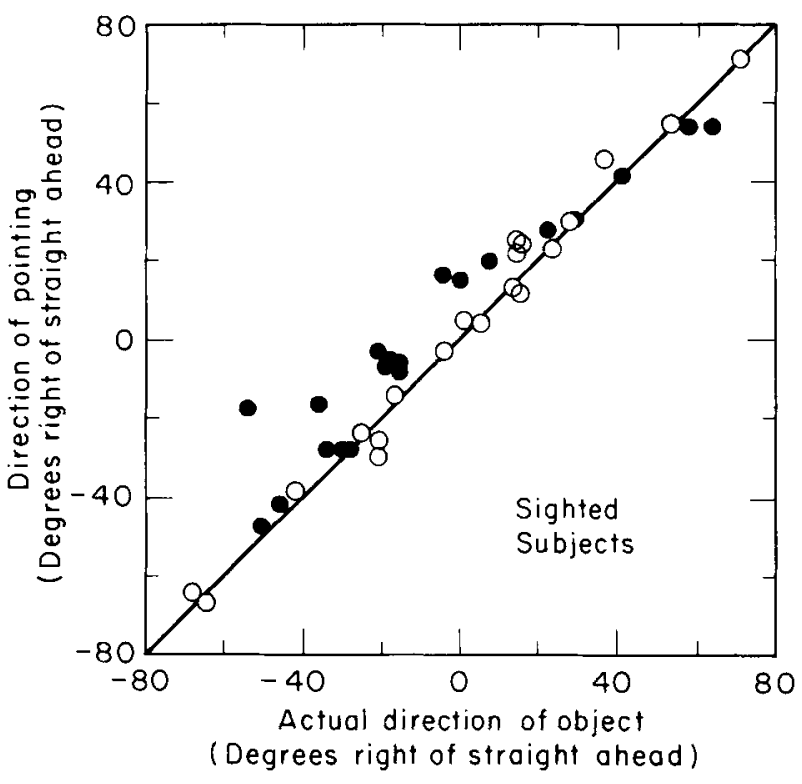

Figure 6. Experiment 3 pointing responses of the sighted subjects, plotted as a function of the actual direction (from the center of the protractor at which the subject stood while responding) to the original location of each test object. Open symbols show responses made from the original station; filled symbols show responses from the new station.

correct direction. The open circles show that responses from the original pointer were much more accurate, but they too show a consistent pattern of errors-one that is different from that demonstrated by the filled circles. From the original station, these subjects pointed too far to the left for objects located on their left and too far to the right for objects located on their right.

These qualitative, as well as quantitative, differences in pointing behavior from the two response stations lend themselves to a simple interpretation that is also consistent with the fact that the updating ability of blind subjects is much greater when replacement is the response mode. This hypothesis is discussed below.

\section{DISCUSSION}

The experiments reported here have examined the ability of early-blind and blindfolded sighted subjects to take their own changed positions into account when recalling the positions of previously examined objects. Our major finding is that the ability of early-blind people to do so depends on the type of behavioral response they are required to use. When asked to point to the locations of objects, the blind subjects in the present study had substantial difficulty in updating, a result consistent with the findings of Rieser et al. $(1982,1986)$. When instructed to replace objects in their original positions on the table, however, the same blind subjects demonstrated a much greater degree of updating skill: accuracy was not significantly affected by whether they were still standing at their original station or had walked part of the way around the table before replacing the objects. The updating ability of the sighted subjects, in contrast, was nearly equivalent under the two sets of experimental conditions.

How are these results to be explained?

One important factor in producing the differences in accuracy between pointing and replacing responses was probably the greater amount of haptic exploration permitted the subjects in Experiment 4 . It will be recalled that when pointing, subjects were instructed not to reach out onto the table again, but to keep their hands in the vicinity of the protractor. When replacing, however, it was by definition necessary that they reach out onto the table again. In doing so, they generally sought and located the edge of the table in one or more places, before deciding where to position the object.

How could this help subjects? No doubt it did so to some degree by allowing them to capitalize on remembered clues, such as the distance of an object from the edge of the table. A subject who recalled that, say, the piece of sponge was a handspan's distance from the edge would probably try to use that information in repositioning it. But, by themselves, facts of this kind would be of very limited value, given that the table was round and featureless. Unless they were able to update, subjects would have no way of knowing from what point on the table's edge the sponge was a handspan's distance. Clues obtained by reaching onto the table could not, in other words, substitute for updating. What they apparently did instead was to allow the blind subjects to demonstrate that updating had already taken place. But how? And why was performance distorted in such a systematic way when these clues were withheld (i.e., in the pointing condition)?

The answer, we believe, is that during testing the blind subjects had a distorted memory of distances on the table. We propose that, soon after these subjects haptically

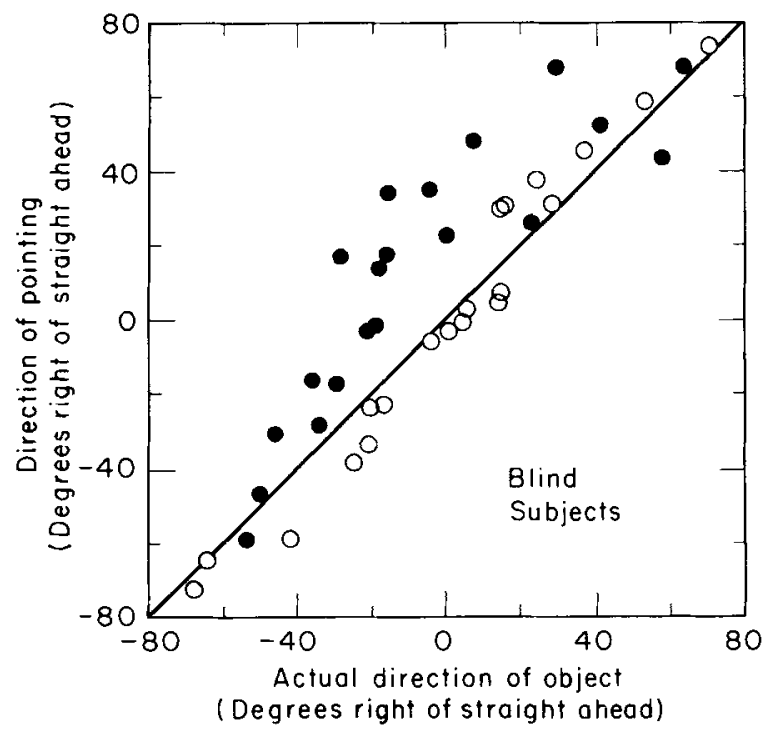

Figure 7. Experiment 3 pointing responses of the blind subjects. Conventions as in Figure 6. 


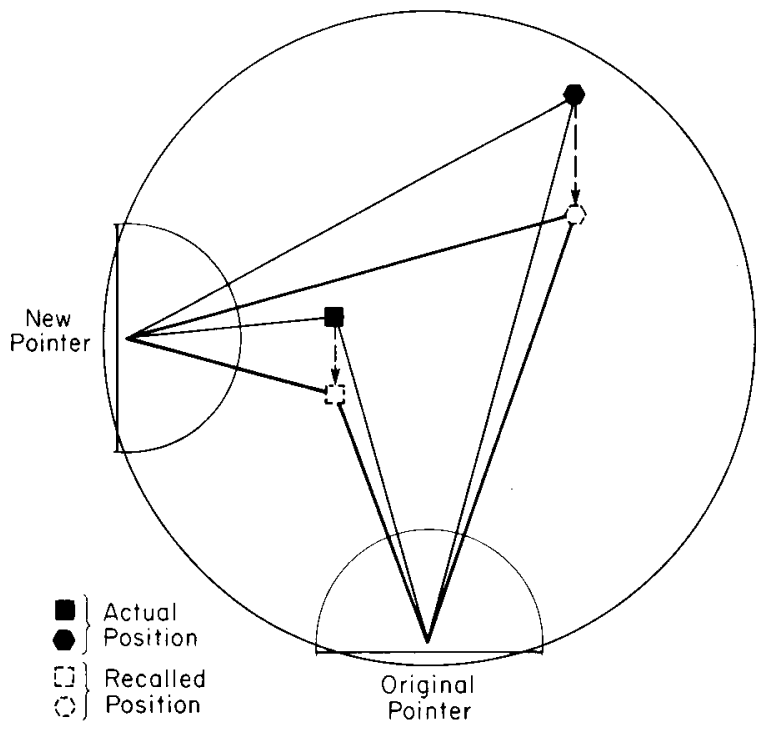

Figure 8. Distortion of spatial memory proposed to explain the patterns of pointing errors obtained in Experiment 3. The filled symbols represent the actual positions of two (hypothetical) objects; the open symbols show their recalled positions. In accordance with the hypothesis offered in the text, forward distances are illustrated as being reduced in memory, compared with left-to-right distances. Heavy lines extending from the pointers to the recalled positions represent the directions in which subjects point during testing; the thin lines show the directions in which an ideal subject would point. This model predicts that pointing errors will usually be larger from the new pointer than from the old.

examined objects during the learning phase of a run, their memory for the locations of those objects underwent a systematic transformation, with recalled fore-and-aft distances shrinking relative to side-to-side distances. Figure 8 illustrates this hypothesis. The filled symbols show the true positions of two (hypothetical) objects on the table; shortly after being examined, their recalled positions shift to the positions indicated by the open symbols. Although we consider it likely that the subject's memory for the dimensions of the table itself undergo a corresponding change, for the sake of simplicity this is not represented in the figure.

The solid lines in the figure illustrate the effect of these hypothesized distortions on pointing responses. The thin lines emanating from the original pointer show the correct directions to the objects; the heavy lines show the directions in which the subject actually does point. Note that leftward errors are made for objects on the left and rightward errors are made for objects on the right; note also that these errors are not very large. When the subject moves to the new pointer, however, the objects will, in memory, be located to the right of their true positions. The subject's errors will therefore all be rightward ones, and will generally be larger than those made from the original pointer. Note that this explanation of the pattern of errors shown in Figure 7 is consistent with, and in fact depends on, good updating ability.
The fact that the sighted subjects consistently pointed somewhat to the right of the correct direction from the new pointer suggests that they may be vulnerable to the same distortion of memory as the blind subjects, but to a lesser extent. Neither group showed the effects of this distortion in the replacement condition, because there they were allowed to reexamine the table and (we believe) correct their faulty memories of its dimensions.

In summary, our hypothesis is that systematic distortions occur while spatial information, derived from haptic examination of the table, is being encoded and committed to memory by the subjects, especially the blind subjects, but that little or no additional distortion occurs, for either group, during the subsequent cognitive operation of updating. Although distortions of haptically perceived spatial extent have been reported by others (Cheng, 1968; Davidon \& Cheng, 1964; Reid, 1954), sometimes for blind as well as sighted subjects (Lederman, Klatzky, \& Barber, 1985; Liddle \& Foss, 1963), the data presented here suggest distortions that are memoric, rather than perceptual, in that they are abolished (as shown by accurate replacing performance) by reexamination of the stimulus field. Perhaps these distortions are the result of haptic spatial biases, analogous to those that operate in the visual modality (e.g., the specific distance tendency [Gogel, 1969]). Such tendencies might be held in abeyance during haptic exploration of the environment, but come into play once subjects have to rely on memory for their knowledge of layout. It remains to be determined in future research whether such haptic biases exist, and if so, whether their strength depends on the extent of a person's previous visual experience.

In their study of updating within a much larger experimental space, Rieser et al. (1986) uncovered patterns of pointing errors that were, in some cases, similar to the patterns we have reported here. They interpreted their data to mean that when subjects responded from the new pointer, they misjudged either their position or the direction they were facing. We have interpreted the data of the present study somewhat differently. We hypothesize that subjects misjudge the dimensions of the stimulus field: the fact that blind subjects made consistent pointing errors even before they left the original pointer favors this interpretation (see Figure 7). However, the two views have many points of similarity, and can probably be reconciled by future experiments. In particular, it would be desirable to obtain data from the same subjects in both large-scale and small-scale environments, to determine whether the errors of a given individual were qualitatively similar in the two situations. Such a comparison might yield insights into the mechanisms underlying individual subjects' spatial judgments, whether accurate or distorted.

\section{REFERENCES}

Carpenter, P. A., \& Eisenberg, P. (1978). Mental rotation and the frame of reference in blind and sighted individuals. Perception \& Psychophysics, 23, 117-124. 
Cheng, M.-F. (1968). Tactile-kinesthetic perception of length. American Journal of Psychology, 81, 74-82.

Davidon, R. S., \& Cheng, M.-F. H. (1964). Apparent distance in a horizontal plane with tactile-kinesthetic stimuli. Quarterly Journal of Experimental Psychology, 16, 277-281.

GoGEL, W. C. (1969). The sensing of retinal size. Vision Research, 9, 3-24.

Holuss, M. (1986). Haptic mental rotation: More consistent in blind subjects? Journal of Visual Impaiment \& Blindness, 80, 950-952.

Lederman, S. J., Klatzky, R. L., \& Barber, P. O. (1985). Spatial and movement-based heuristics for encoding pattern information through touch. Journal of Experimental Psychology: General, 114, 33-49.

LidDLE, D., \& Foss, B. M. (1963). The tactile perception of size: Some relationships with distance and direction. Quarterly Journal of Experimental Psychology, 15, 217-219.

MARMOR, G. S., \& ZABACK, L. A. (1976). Mental rotation by the blind: Does mental motation depend on visual imagery? Journal of Experimental Psychology: Human Perception \& Performance, 2, 515-521.

REID, R. L. (1954). An illusion of movement complementary to the horizontal-vertical illusion. Quarterly Journal of Experimental Psychology, 6, 107-111.

RIEser, J. J., GuTH, D. A., \& Hill, E. W. (1982). Mental processes mediating independent travel: Implications for orientation and mobility. Journal of Visual Impairment \& Blindness, 76, 213-218.

Rieser, J. J., GuTh, D. A., \& Hill, E. W. (1986). Sensitivity to perspective structure while walking without vision. Perception, 15 , 173-188.

Warken, D. H., Anooshian, L. J., \& Bollinger, J. G. (1973). Early vs. late blindness: The role of early vision in spatial behavior. American Foundation for the Blind Research Bulletin, 26, 151-170.

Witkin, H. A., Birnbaum, J., Lomonaco, S., Lehr, S., \& Herman, J. L. (1968). Cognitive patterning in congenitally totally blind children. Child Development, 39, 767-786.

WorChel, P. (1951). Space perception and orientation in the blind. Psychological Monographs: General \& Applied, 65(15, Whole No. 332).

(Manuscript received May 11, 1987;

revision accepted for publication October 1, 1987.) 